\title{
A comparison of the assessment of health status between CCQ and CAT in a Chinese COPD clinical population: a cross-sectional analysis
}

This article was published in the following Dove Press journal: International Journal of COPD

\author{
Zijing Zhou ${ }^{1-3}$ \\ Aiyuan Zhoul-3 \\ Yiyang Zhaol-3 \\ Jiaxi Duan ${ }^{1-3}$ \\ Ping Chen ${ }^{1-3}$
}

'Department of Respiratory Medicine,

The Second Xiangya Hospital,

Central South University, Changsha,

China; ${ }^{2}$ Research Unit of Respiratory

Disease, Central South University,

Changsha, China; ${ }^{3}$ Diagnosis and

Treatment Center of Respiratory

Disease, Central South University,

Changsha, China
Correspondence: Ping Chen

Department of Respiratory Medicine, The Second Xiangya Hospital, Central South University, I 39 Renmin Middle Road, Changsha, Hunan 4I00II, China Email pingchen073।@csu.edu.cn
Background: Both the COPD Assessment Test (CAT) and the Clinical COPD Questionnaire (CCQ) were recommended as comprehensive symptom measures by the Global Initiative for Chronic Obstructive Lung disease. The main objective of this work was to compare the evaluation of symptom severity with the CAT and the CCQ and find a cutoff value of the CCQ for the Chinese clinical population.

Methods: Patients diagnosed with stable COPD in the outpatient department who completed the CAT and CCQ were enrolled from November 2015 to December 2016. Scores of 0-10, 11-20, 21-30, and 31-40 represent low, medium, high, and very high impact level, respectively, by CAT. Scores of the CCQ can be considered as acceptable $(\mathrm{CCQ}<1)$, acceptable for moderate disease $(1 \leq \mathrm{CCQ}<2)$, instable-severe limited $(2 \leq \mathrm{CCQ}<3)$, and very instable-very severe limited (CCQ $\geq 3)$.

Results: According to the CAT, only $20.4 \%$ of patients belonged to the high $(21 \leq \mathrm{CAT} \leq 30)$ and very high $(31 \leq \mathrm{CAT} \leq 40)$ impact levels, which were statistically lower than those of the CCQ, which classified over half of the population (51.1\%) into the instable-severe limited $(2 \leq \mathrm{CCQ}<3)$ and very instable-very severe limited $(\mathrm{CCQ} \geq 3)$ categories $(P<0.001)$. The kappa of agreement for the symptom groups by CAT and CCQ (cutoff point 1.5) was 0.495 , but only slight agreement (0.144) was found between the CAT and CCQ with the cutoff point of 1.0. The CAT and the total CCQ had a strong correlation (rho=0.776, $P<0.01$ ). The CCQ 1.0 and 1.5 corresponded to CAT 4.2 and 9.7, respectively. The CAT 10.0 was equivalent to 1.53 of the CCQ. In addition, with higher scores of the CAT and CCQ, subjects displayed more impairment in lung function, higher levels on modified Medical Research Council, and higher exacerbation rates in the last year $(P<0.001)$. Similarly, patients with more exacerbations presented worse scores on the CAT and total CCQ as well as its 3 domains $(P<0.001)$.

Conclusion: Compared with the CAT, the CCQ was more likely to classify the patients into more severe categories, and 1.5 might be a better cutoff point for the CCQ than 1.0. Both the CAT and the overall CCQ with its 3 domains were able to discriminate between groups of patients that differ in COPD severity.

Keywords: chronic obstructive pulmonary disease, COPD, the Clinical COPD Questionnaire, CCQ, the COPD Assessment Test, CAT

\section{Introduction}

COPD is a leading cause of morbidity and mortality throughout the world and creates an enormous social and economic burden on the entire population. ${ }^{1}$ The overall impact of COPD on individuals is multifaceted and it causes impairment not only in the lungs but also in other organs, and even in psychological conditions, which 
result in multiple symptomatic effects and poorer quality of life. Therefore, the Global Initiative of Chronic Obstructive Lung Disease (GOLD) proposed a novel multidimensional assessment since 2011 that included the level of health status and the risk of experiencing an exacerbation in addition to lung function. ${ }^{1}$

Both the COPD Assessment Test (CAT) and the Clinical COPD Questionnaire (CCQ) were recommended as comprehensive symptom measures by the GOLD. ${ }^{2}$ The CAT was developed by Jones et $\mathrm{al}^{3}$ in 2009 and consists of 8 items related to respiratory disorders: cough, phlegm, chest tightness, breathlessness, activity limitation at home, confidence in leaving home, sleep, and energy. The CCQ was developed and validated in 2003 and has 3 domains (symptoms, mental, and functional), with items scored from 0 to $6 .^{4}$ The CAT and CCQ have some common features: both of these 2 tools contain items of cough, phlegm, and breathlessness; in addition, higher scores correspond with a worse health status. However, the tools also differ in some details: compared with the CAT, the CCQ has a more detailed assessment of physical and mental activities whereas it does not focus on the patient's chest tightness and sleep quality. Moreover, the cutoff for the CCQ is still controversial: GOLD 2013 suggested a cutoff point of $>1$ for the CCQ 2; however, Kon et $\mathrm{al}^{5}$ found that 1.5 may be a more appropriate cutoff point for classifying symptom levels.

To the best of our knowledge, there have been no studies focused on the utility of the CCQ in a clinical population from mainland China. The main objective of this work was to compare the evaluation of symptom severity between the CAT and the CCQ and to find a cutoff value of the CCQ for the Chinese clinical population. In addition, we also examined the correlation between the CAT, the CCQ, the modified Medical Research Council (mMRC) questionnaire, lung function, and exacerbation history.

\section{Methods}

\section{Study design and subjects}

This was an observational, cross-sectional, cohort study that aimed to compare the CAT and CCQ in outpatients with stable COPD from the Second Xiangya Hospital of Central South University (registration number: ChiCTR-OOC15007352). All participants in the study provided a written informed consent. The study was approved by the Second Xiangya Hospital of Central South University institutional review board (Hunan, China).

Patients diagnosed with stable COPD in the outpatient department were enrolled from November 2015 to December 2016. All patients were aged $>40$ years. Patients were also required to have the ability to undertake spirometry with a ratio of the forced expiratory volume in $1 \mathrm{~s}$ to the forced vital capacity $\left(\mathrm{FEV}_{1} / \mathrm{FVC}\right)$ lower than 0.70 after bronchodilation. ${ }^{2}$ Patients who refused to complete questionnaires and those with asthma, lung cancer or other lung disease under active treatment, such as tuberculosis or pneumonia, were excluded.

\section{Data collection and definition of exacerbation history}

Information on age, sex, smoking status, number of exacerbations in the previous year, height, weight, lung function, and the scores of the mMRC, CAT, and CCQ were recorded. Exacerbations in the previous year were assessed according to event-based definition: worsening or new onset of any respiratory symptoms (cough, sputum volume or purulence, wheezing, or dyspnea) for at least 3 days that leads to any 1 of following situations: 1) requiring prescription change: increase in the dose or/and frequency of bronchodilator at home or requiring treatment with oral corticosteroids or/and antibiotics under the guidance of a physician in the outpatient department; 2) requiring a hospitalization and diagnosed with acute exacerbations of COPD (AECOPD); and 3) requiring an emergency room visit and diagnosed with AECOPD.

$\mathrm{mMRC}$

The mMRC dyspnea scale is used for grading the effect of breathlessness (dyspnea) on daily activities. Patients select 1 of 5 grades that most closely corresponds to their level of impairment, from grade 0 (least severe breathlessness) to grade 4 (most severe breathlessness).

\section{CAT}

The CAT consists of 8 items, with scores ranging from 0 to $5(0=$ no impairment, $5=$ greatest impairment $)$. An overall score is calculated by simply adding the points of the 8 questions ranging from 0 to 40 , with higher scores indicating more severe health status impairment or a poorer control of COPD. Four scores of 0-10, 11-20, 21-30, and 31-40 represent low, medium, high, and very high impact level, respectively. ${ }^{6}$

\section{CCQ}

The CCQ contains 10 items, divided into 3 domains (symptoms, functional, and mental state). The total score is calculated by adding all answers and dividing them by the number of questions, that is, by 10 . Similarly, domains are calculated by adding each domain's answers and dividing by 4 for the symptom and the functional domain and by 2 for the mental domain. ${ }^{4}$ The CCQ results can be interpreted as: acceptable 
$(\mathrm{CCQ}<1)$; acceptable for moderate disease $(1 \leq \mathrm{CCQ}<2)$; instable-severe limited $(2 \leq \mathrm{CCQ}<3)$; and very instable-very severe limited $(\mathrm{CCQ} \geq 3){ }^{6}$

\section{Statistical analysis}

All data were input and analyzed using SPSS 20.0 software. The chi-square, analysis of variance, and Mann-Whitney $U$-test were used as appropriate to compare differences between groups. Kappa coefficient was used to analyze the agreement between the CAT and the CCQ. The kappa value was based on the literature, in which $k<0.00$ is "poor," $0<k<0.02$ is "slight," $0.21<k<0.40$ is "fair," $0.41<k<0.60$ is "moderate," $0.61<k<0.80$ is "substantial," $0.81<k<1.00$ is "almost perfect," and $k=1$ is "perfect" agreement. ${ }^{7}$ The correlation between health status questionnaires (CAT and $\mathrm{CCQ}$ ), lung function, and exacerbation frequency in the previous year and the mMRC dyspnea scale was calculated by the Spearman rank correlation coefficient (rho). Differences were considered statistically significant when $P<0.05$.

\section{Results}

\section{Study population}

A total of 372 patients diagnosed with COPD from the outpatient department completed all the 3 symptom-evaluation questionnaires (mMRC, CAT, and CCQ) and met the inclusion criteria. Basic characteristics of participants are shown in Table 1 . The majority of patients were male, with an average age of 62.2 years. The mean body mass index was $22.3 \mathrm{~kg} / \mathrm{m}^{2}$. More than half of the subjects had experienced at least 1 exacerbation in the last 12 months, and a quarter of them had 2 or more. The mean $\mathrm{FEV}_{1} \%$ predicted was 48.6. Based on the GOLD 2007 classification, $8.1 \%$ patients had mild COPD (grade 1), 32.8\% moderate COPD (grade 2), $46.2 \%$ had severe COPD (grade 3 ), and $12.9 \%$ had very severe COPD (grade 4 ). The mean (SD) scores of the mMRC, CAT, and CCQ were $1.9(0.9), 15.4(6.0)$, and $2.0(0.7)$, respectively.

\section{Differences in the assessment of health status between CCQ and CAT}

According to the CAT, only $20.4 \%$ of patients belonged to the high $(21 \leq \mathrm{CAT} \leq 30)$ and very high $(31 \leq \mathrm{CAT} \leq 40)$ impact levels, which were statistically lower than those of the CCQ, which classified over half of the population $(51.1 \%)$ into the instable-severe limited $(2 \leq \mathrm{CCQ}<3)$ and very instablevery severe limited $(\mathrm{CCQ} \geq 3)$ categories correspondingly $(P<0.001$, data not shown).

The CCQ cutoff point of 1.0 classified $97.3 \%$ of patients into the high symptom group (groups B and D), which was
Table I Demographic and clinical characteristics of participants with COPD

\begin{tabular}{|c|c|}
\hline Characteristics & $\begin{array}{l}\text { All patients } \\
(n=372)\end{array}$ \\
\hline Age (years) & $62.2 \pm 8.3$ \\
\hline \multicolumn{2}{|l|}{ Gender } \\
\hline Male & $319(85.8 \%)$ \\
\hline Female & $53(14.2 \%)$ \\
\hline BMI $\left(\mathrm{kg} / \mathrm{m}^{2}\right)$ & $22.3 \pm 3.5$ \\
\hline Smoking pack years & $40.0 \pm 32.2$ \\
\hline \multicolumn{2}{|l|}{ Exacerbations in the last 12 months } \\
\hline 0 & I 75 (47.0\%) \\
\hline I & $104(28.0 \%)$ \\
\hline$\geq 2$ & $93(25.0 \%)$ \\
\hline $\mathrm{FEV}_{1} / \mathrm{FVC}$ & $0.44 \pm 0.13$ \\
\hline $\mathrm{FEV}$ \% predicted & $48.6 \pm 18.9$ \\
\hline $\mathrm{mMRC}$ & $1.9 \pm 0.9$ \\
\hline CAT & $15.4 \pm 6.0$ \\
\hline Mild $(0-10)$ & $85(22.8 \%)$ \\
\hline Medium (I I-20) & $211(56.7 \%)$ \\
\hline High $(2 \mathrm{I}-30)$ & $72(19.4 \%)$ \\
\hline Very high $(31-40)$ & $4(1.1 \%)$ \\
\hline $\mathrm{CCQ}$ & $2.0 \pm 0.7$ \\
\hline Acceptable $(\mathrm{CCQ}<\mathrm{I})$ & $10(2.7 \%)$ \\
\hline Acceptable for moderate disease $(I \leq C \mathrm{CQ}<2)$ & $172(46.2 \%)$ \\
\hline Instable-severe limited $(2 \leq \mathrm{CCQ}<3)$ & $156(41.9 \%)$ \\
\hline Very instable-very severe limited $(\mathrm{CCQ} \geq 3)$ & $34(9.2 \%)$ \\
\hline \multicolumn{2}{|l|}{ GOLD grades } \\
\hline I: Mild & $30(8.1 \%)$ \\
\hline 2: Moderate & $122(32.8 \%)$ \\
\hline 3: Severe & I 72 (46.2\%) \\
\hline 4: Very severe & $48(12.9 \%)$ \\
\hline
\end{tabular}

Note: Data presented as mean \pm SD or $\mathrm{n}(\%)$.

Abbreviations: BMI, body mass index; CAT, COPD Assessment Test; CCQ, Clinical COPD Questionnaire; FEV , forced expiratory volume in I second; FVC, forced vital capacity; GOLD, global initiative for chronic obstructive lung disease; mMRC, modified Medical Research Council dyspnea score.

statistically higher than that of the CAT $(82.3 \%)$ and CCQ with a cutoff point of $1.5(78.0 \%)$. No significant difference was observed in the proportion of subjects who had more symptoms between the CAT and CCQ with the cutoff point of 1.5 (Figure 1). The kappa of agreement for the symptom groups by CAT and CCQ (cutoff point 1.5) was 0.495 , suggesting moderate agreement (Table 2), but slight agreement (0.144) was found between the CAT and CCQ with the cutoff point of 1.0 (Table 3).

\section{$\mathrm{FEV}, / \mathrm{FVC}, \mathrm{FEV}$ \% predicted, exacerbation frequency, and $\mathrm{mMRC}$ in different CAT and CCQ categories}

Because there were only 4 patients in the very high $(31 \leq \mathrm{CAT} \leq 40)$ group of CAT, we compared only lung function, exacerbation frequency, and mMRC between mild, high, and very high CAT categories. As shown in 


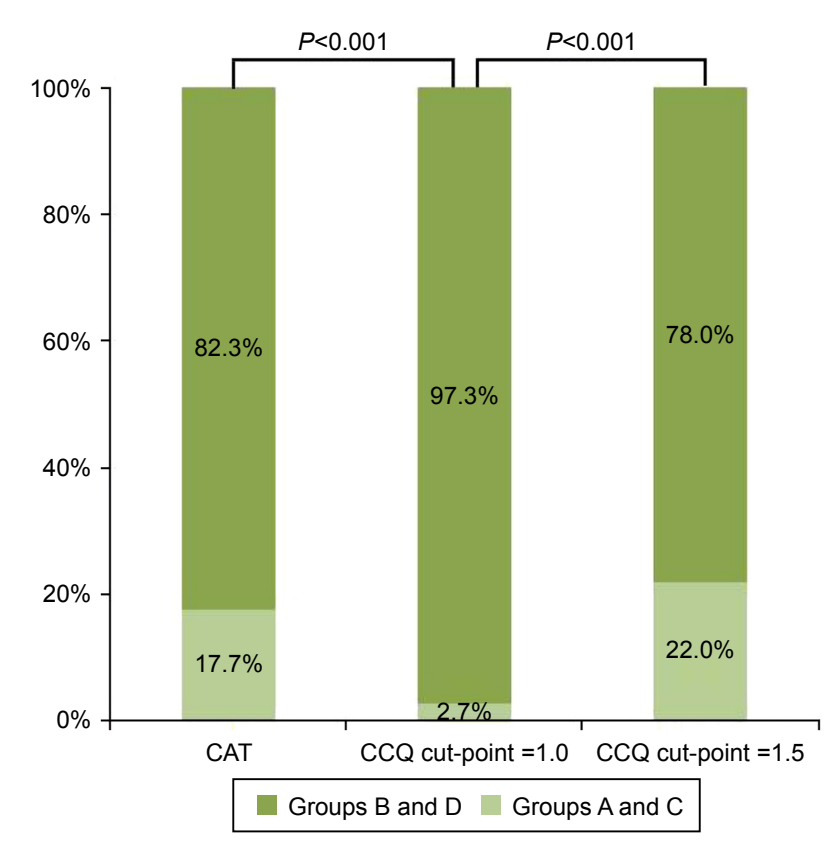

Figure I Differences in classification of symptom group using CAT and CCQ. Abbreviations: CAT, COPD Assessment Test; CCQ, Clinical COPD Questionnaire.

Tables 4 and 5, with higher impact levels of the CAT or increased severity of the CCQ, both exacerbation frequency and the mMRC score were markedly increased $(P<0.001)$, whereas $\mathrm{FEV}_{1} / \mathrm{FVC}$ and $\mathrm{FEV}_{1} \%$ predicted significantly decreased $(P<0.001)$. However, no statistical differences were found in $\mathrm{FEV}_{1} / \mathrm{FVC}$ and $\mathrm{FEV}_{1} \%$ predicted between patients in the acceptable $(\mathrm{CCQ}<1)$ and acceptable for moderate disease $(1 \leq \mathrm{CCQ}<2)$ groups $(P>0.05)$. Moreover, subjects from the instable-severe limited and very instablevery severe limited group by the CCQ also had no difference in $\mathrm{FEV}_{1} / \mathrm{FVC}(P>0.05)$.

\section{Score of CAT and CCQ in different GOLD grades}

Both the CAT and the CCQ total score increased with the COPD severity assessed by GOLD grades (Table 6). The total CAT and CCQ scores were significantly higher in subjects

Table 2 Kappa of agreement for the symptom groups by CAT and CCQ (cutpoint =I.5)

\begin{tabular}{llll}
\hline CCQ & CAT & & \\
\cline { 2 - 4 } cutpoint $=1.5$ & Groups A and C & Groups B and D & Total \\
\hline Groups A and C & 44 & 38 & 82 \\
Groups B and D & 22 & 268 & 290 \\
Total & 66 & 306 & 372 \\
\hline
\end{tabular}

Note: Kappa $=0.495$, moderate agreement.

Abbreviations: CAT, COPD Assessment Test; CCQ, Clinical COPD Questionnaire.
Table 3 Kappa of agreement for the symptom groups by CAT and CCQ (cutpoint $=I .0$ )

\begin{tabular}{llll}
\hline CCQ & CAT \\
cutpoint $=\mathbf{I . 0}$ & Groups A and C & Groups B and D & Total \\
\hline Groups A and C & 7 & 3 & 10 \\
Groups B and D & 59 & 303 & 362 \\
Total & 66 & 306 & 372 \\
\hline
\end{tabular}

Note: Kappa $=0.144$, slight agreement.

Abbreviations: CAT, COPD Assessment Test; CCQ, Clinical COPD Questionnaire.

from grade 2 vs 3 and those from grade 3 vs 4; however, no statistical differences were observed between grades 1 and 2. We also compared the 3 domains of the CCQ among all GOLD grades. Participants classified as grades 1, 2, and 3 had no statistically different scores in the CCQ symptom and mental domain, but patients in grades 3-4 scored significantly higher than those from grades 1-2 in the CCQ functional domain. Fewer exacerbations in the last 12 months were reported among patients in grade 1 compared with grades 3-4 $(P<0.05)$, whereas there was no statistical difference in the number of exacerbations between grades $1-2$, grades $2-3$, and grades 3-4 (Table 6).

\section{Score of CAT and CCQ by exacerbation frequencies in the previous year}

According to exacerbation frequencies, we classified patients into 3 levels: 0 exacerbation, 1 exacerbation, and 2 or more exacerbations. Of 372 subjects, 175 (47\%) had no exacerbations in the last year. The mean CAT score was 12.69 (5.11), and the average score of total CCQ, symptom domain, functional domain and mental domain were $1.70(0.60), 1.84$ (0.68), 1.60 (0.69), and 1.41 (1.09), respectively. All the results of health status questionnaires of patients with no exacerbations were significantly lower than those of participants who had 1 exacerbation. A similar trend was also observed between patients who reported 1 exacerbation and those had at least 2 exacerbations in the last 12 months; however, there was no statistical difference in $\mathrm{FEV}_{1} \%$ predicted between these 2 groups, despite the fact that subjects who never had exacerbations had a statistically higher value of $\mathrm{FEV}_{1} \%$ predicted than those who reported at least 1 exacerbation (Table 7).

\section{Distribution of CAT and CCQ levels into different exacerbation frequencies}

Figures 2 and 3 show the distribution of CAT and CCQ levels into different exacerbation frequencies. With the higher level of CAT and CCQ (worse health status), the proportion of 
Table 4 FEV $/$ FVC, FEV \% predicted, exacerbation frequency and mMRC in different CAT categories

\begin{tabular}{|c|c|c|c|c|}
\hline Variables & $\begin{array}{l}\text { Mild } \\
0 \leq \text { CAT } \leq 10\end{array}$ & $\begin{array}{l}\text { Medium } \\
\mid \mathrm{I} \leq \mathrm{CAT} \leq \mathbf{2 0}\end{array}$ & $\begin{array}{l}\text { High } \\
2 \mathrm{I} \leq \mathrm{CAT} \leq 30\end{array}$ & P-value \\
\hline $\mathrm{FEV}_{1} / \mathrm{FVC}$ & $0.47 \pm 0.13^{\#, \Delta}$ & $0.44 \pm 0.12 *$, & $0.39 \pm 0.14^{*, \#}$ & $<0.001$ \\
\hline $\mathrm{FEV} \% \%$ predicted & $54.73 \pm 20.9 l^{\#, \Delta}$ & $48.28 \pm 17.48^{*, 4}$ & $42.72 \pm 18.79 *, \#$ & $<0.001$ \\
\hline Exacerbation frequency in the last year & $0.28 \pm 0.59^{\#, \Delta}$ & $0.92 \pm 1.22 *, \Delta$ & $1.86 \pm 1.37^{*, \#}$ & $<0.001$ \\
\hline $\mathrm{mMRC}$ & $1.18 \pm 0.69^{\#,}$ & $1.79 \pm 0.83^{*, 4}$ & $2.50 \pm 0.7 \mid *, \#$ & $<0.001$ \\
\hline
\end{tabular}

Notes: $* P<0.05$ vs $0 \leq \mathrm{CAT} \leq 10 ;{ }^{*} P<0.05$ vs $I \mathrm{I} \leq \mathrm{CAT} \leq 20 ;{ }^{\wedge} P<0.05$ vs $2 \mathrm{I} \leq \mathrm{CAT} \leq 30$.

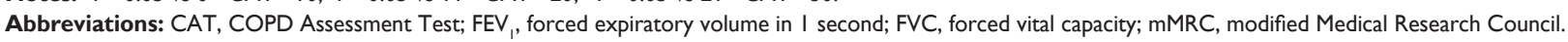

Table 5 FEV /FVC, FEV,\% predicted, exacerbation frequency, and mMRC in different CCQ categories

\begin{tabular}{|c|c|c|c|c|c|}
\hline Variables & $\begin{array}{l}\text { Acceptable } \\
\text { cce }<\text { I }\end{array}$ & $\begin{array}{l}\text { Acceptable for } \\
\text { moderate disease } \\
\mathrm{I} \leq \mathrm{CCQ}<\mathbf{2}\end{array}$ & $\begin{array}{l}\text { Instable-severe } \\
\text { limited } \\
\mathbf{2} \leq \mathrm{CCQ}<\mathbf{3}\end{array}$ & $\begin{array}{l}\text { Very instable-very } \\
\text { severe limited } \\
\mathrm{CCQ} \geq 3\end{array}$ & $P$-value \\
\hline $\mathrm{FEV}_{1} / \mathrm{FVC}$ & $59.90 \pm 15.82^{\wedge, \pi}$ & $46.19 \pm 12.11^{\mathbf{4}, \pi}$ & $42.05 \pm 12.33^{*, \#}$ & $37.5 \mathrm{I} \pm \mathrm{II} .52^{*, \#}$ & $<0.001$ \\
\hline $\mathrm{FEV}, \%$ predicted & $62.53 \pm 21.06^{4, \pi}$ & $51.53 \pm 18.10^{4, \pi}$ & $46.65 \pm 18.94^{*, \#, \pi}$ & $38.34 \pm 17.39 *, \#, 4$ & $<0.001$ \\
\hline Exacerbation frequency in the last year & $0.10 \pm 0.32^{\#, \Delta, \pi}$ & $0.49 \pm 0.98 *, 4 \pi$ & $1.26 \pm 1.22 * \#$ & $2.44 \pm 1.48^{*, \#, \Delta}$ & $<0.001$ \\
\hline $\mathrm{mMRC}$ & $0.70 \pm 0.48^{\#, \Delta, \pi}$ & I. $54 \pm 0.79 *, \triangle, \pi$ & $2.19 \pm 0.73^{*, \#, \pi}$ & $2.68 \pm 0.77^{*, \#, \Delta}$ & $<0.001$ \\
\hline
\end{tabular}

Notes: $*^{*}<0.05$ vs $C C Q<1 ;{ }^{P} P<0.05$ vs $I \leq C C Q<2 ;{ }^{\wedge} P<0.05$ vs $2 \leq C C Q<3 ;{ }^{\top} P<0.05$ vs $C C Q \geq 3$.

Abbreviations: CCQ, clinical COPD questionnaire; $\mathrm{FEV}_{1}$, forced expiratory volume in I second; FVC, forced vital capacity; mMRC, modified Medical Research Council dyspnea score.

Table 6 Score of CAT and CCQ by GOLD grades

\begin{tabular}{|c|c|c|c|c|c|}
\hline Variables & Grade $I, n=30$ & Grade 2, n= 122 & Grade 3, $n=172$ & Grade 4, n=48 & $P$-value \\
\hline CAT & $13.80 \pm 6.27 \pi$ & $13.89 \pm 5.28^{4}, \pi$ & $|5.56 \pm 5.6|^{\#, \pi}$ & $19.77 \pm 6.70^{*, \#, \Delta}$ & $<0.001$ \\
\hline CCQ total & $1.76 \pm 0.70^{4} \pi$ & $1.85 \pm\left. 0.6\right|^{4, \pi}$ & $2.06 \pm 0.69 *, \#, \pi$ & $2.5 \mathrm{I} \pm 0.93^{*, \#, \Delta}$ & $<0.001$ \\
\hline CCQ symptom & $2.02 \pm 0.84 \pi$ & $2.06 \pm 0.76 \pi$ & $2.16 \pm 0.80 \pi$ & $2.50 \pm 1.03^{*, \#, \Delta}$ & $=0.013$ \\
\hline CCQ function & $1.53 \pm 0.8 I^{\Delta, \pi}$ & I. $70 \pm 0.68^{4, \pi}$ & $1.97 \pm 0.76^{* \# \#}$ & $2.53 \pm 1.02^{*, \# \Delta}$ & $<0.001$ \\
\hline CCQ mental & $1.68 \pm 1.05$ & $1.62 \pm 1.09 \pi$ & $1.86 \pm 1.14$ & $2.18 \pm 1.42^{\#}$ & $=0.033$ \\
\hline Exacerbation frequency & $0.37 \pm 0.67^{4, \pi}$ & $0.79 \pm 1.20 \pi$ & $1.06 \pm 1.31 *$ & $1.65 \pm\left. 1.4\right|^{*, \#}$ & $<0.001$ \\
\hline
\end{tabular}

Notes: $* P<0.05$ vs Grade I; ${ }^{\#} P<0.05$ vs Grade $2 ;{ }^{\wedge} P<0.05$ vs Grade $3 ;{ }^{\Uparrow} P<0.05$ vs Grade 4.

Abbreviations: CAT, COPD Assessment Test; CCQ, clinical COPD questionnaire; GOLD, Global Initiative for Chronic Obstructive Lung Disease.

Table 7 Score of CAT and CCQ by exacerbation frequencies

\begin{tabular}{|c|c|c|c|c|}
\hline \multirow[t]{2}{*}{ Variables } & \multicolumn{3}{|c|}{ Exacerbation frequency in the last 12 months } & \multirow[t]{2}{*}{$P$-value } \\
\hline & $A E=0, n=175$ & $A E=I, n=104$ & $A E \geq 2, n=93$ & \\
\hline CAT & $12.69 \pm 5.1 I^{\#, \Delta}$ & $|5.6| \pm 4.76^{*, \Delta}$ & $20.33 \pm 5.57^{* \#}$ & $<0.001$ \\
\hline CCQ total & $1.70 \pm 0.60^{\#, \Delta}$ & $2.02 \pm 0.47^{*,}$ & $2.64 \pm 0.8 I^{* \#}$ & $<0.001$ \\
\hline CCQ symptom & $1.84 \pm 0.68^{\#, \Delta}$ & $2.13 \pm 0.64^{*, \Delta}$ & $2.78 \pm 0.92^{*, \#}$ & $<0.001$ \\
\hline CCQ function & $1.60 \pm 0.69^{\#, \Delta}$ & $1.91 \pm 0.60^{*, 4}$ & $2.52 \pm 0.94^{* \# \#}$ & $<0.001$ \\
\hline CCQ mental & $1.4 \mathrm{I} \pm 1.09^{\#, \Delta}$ & $1.96 \pm 0.90 *$ & $2.38 \pm 1.31^{*, \#}$ & $<0.001$ \\
\hline FEV \% predicted & $53.84 \pm 19.32^{\#, \mathbf{\Delta}}$ & $45.37 \pm 18.22 *$ & $42.25 \pm 16.30 *$ & $<0.001$ \\
\hline
\end{tabular}

Notes: $* P<0.05$ vs $A E=0 ;{ }^{*} P<0.05$ vs $A E=1 ;{ }^{\wedge} P<0.05$ vs $A E \geq 2$.

Abbreviations: CAT, COPD Assessment Test; CCQ, clinical COPD questionnaire; FEV 


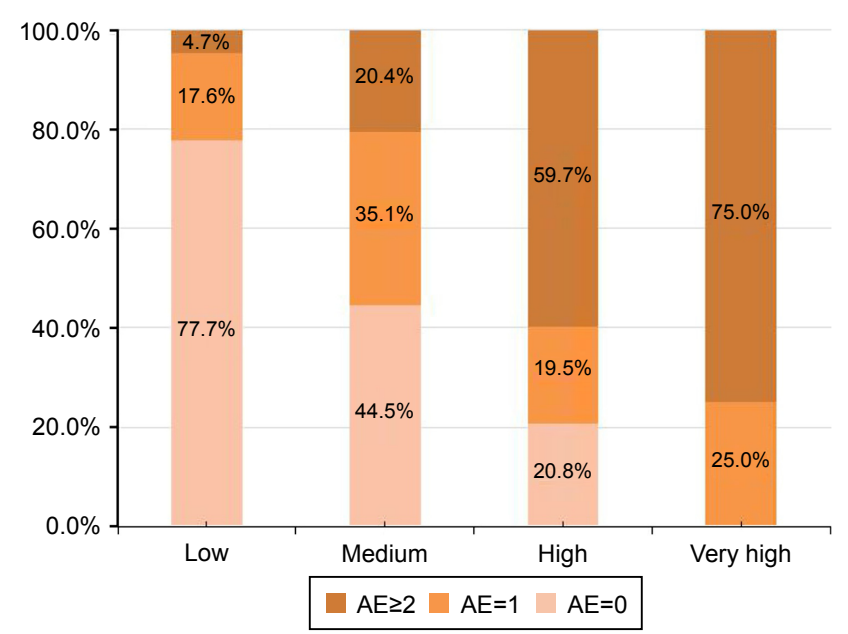

Figure 2 Distribution of CAT impact levels into different exacerbation frequencies. Abbreviation: CAT, COPD Assessment Test.

participants who had no exacerbations in the previous year gradually decreased, but the percentage of subjects who reported at least 2 exacerbations had a completely opposite trend.

Among the subjects with frequent exacerbations $(\geq 2)$, only $2.2 \%$ had a CAT score $<10$ (fewer symptoms), and $5.4 \%$ had a total CCQ score $<1.5$. In addition, no patient had a score of total CCQ $<1.0$ (data not shown).

\section{Spearman rank correlations between CAT and CCQ questionnaires, lung function, exacerbation frequency, and mMRC dyspnea scale}

In comparing $\mathrm{FEV}_{1} \%$ predicted results, the $\mathrm{mMRC}$ and all 3 domains of the CCQ, the CAT, and the total CCQ score

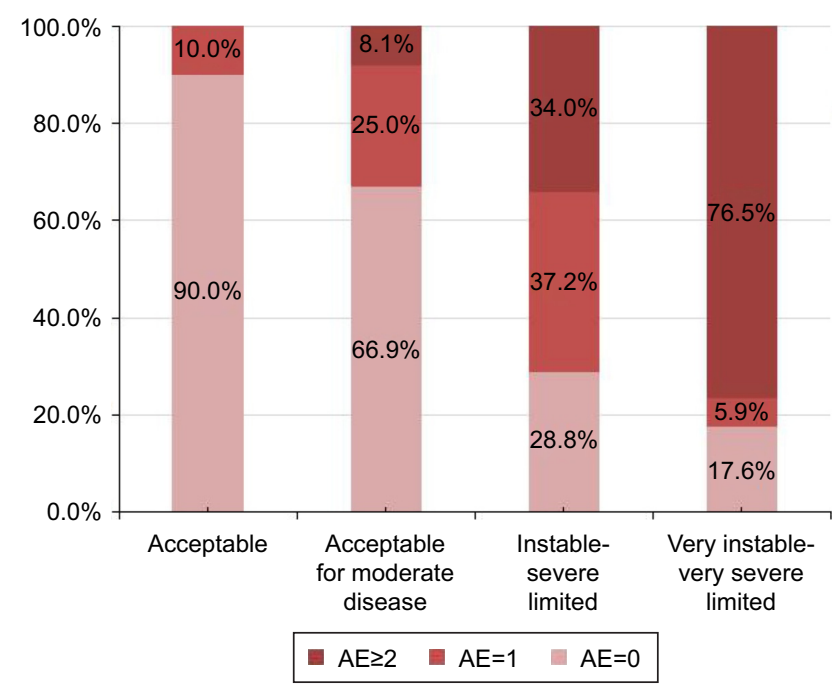

Figure 3 Distribution of CCQ categories into different exacerbation frequencies. Abbreviation: CCQ, clinical COPD questionnaire. had a much better correlation with exacerbation frequency (Table 8). Moreover, the CAT and the total CCQ were significantly correlated with each other (rho=0.776, $P<0.01$ ). The CCQ 1.0 and 1.5 corresponded to CAT 4.2 and 9.7, respectively. The CAT 10.0 was equivalent to 1.53 of the CCQ (Figure 4).

\section{Discussion}

The main findings of this cross-sectional study were as follows: 1) compared with the CAT, the CCQ was more likely to classify the patients into more severe categories, and it seemed that 1.5 rather than 1.0 might be a better cutoff point for the CCQ and 2) both the CAT and the overall CCQ with its 3 domains could discriminate between groups of patients who differ in COPD severity.

The CAT and CCQ results can be categorized into 4 levels: scores of 0-10, 11-20, 21-30, and 31-40 represent low, medium, high, and very high impact level by CAT. Correspondingly, scores of the CCQ can be considered as acceptable $(\mathrm{CCQ}<1)$, acceptable for moderate disease $(1 \leq \mathrm{CCQ}<2)$, instable-severe limited $(2 \leq \mathrm{CCQ}<3)$, and very instable-very severe limited (CCQ $\geq 3) .{ }^{6}$ In this study, $51.1 \%$ of patients had instable-severe limited or very instable-very severe limited disease $(C C Q \geq 2)$, whereas only $20.5 \%$ were in the high-very high group of CAT (CAT $\geq 21)$, which had a statistical difference. This could be explained by the most obvious difference between these 2 instruments, which is that CAT has no corresponding items related to the mental domain of the CCQ. We further observed that 120 subjects who had a score of $\mathrm{CCQ} \geq 2$ were classified into mild-medium impact levels by CAT $(\mathrm{CAT}<20)$, importantly, all 120 subjects had mental impairment, with an average score of $(2.3 \pm 0.9)$ in the mental domain. Evidence has shown that mental diseases, such as depression and anxiety in COPD patients can have significantly deleterious effects, including an increased length of hospitalization and mortality risk..$^{8-10}$ Our work also confirmed that patients with more exacerbations had worse mental status; thus, the management and treatment of mental disease in COPD should be paid much more attention.

A notable finding of this study is that 1.5 is a more appropriate cutoff point for the CCQ than 1.0, at least in the Chinese population. We show that for the CCQ 1.0 and 1.5, CAT is equivalent to 4.2 and 9.7, respectively, and the CAT score of 10.0 corresponds with a score of 1.53 on the CCQ. These results are consistent with Kon's study ${ }^{5}$ Our work also finds that when the cutoff value of the CCQ is 1.5 , it has a better agreement with the CAT than that of 1.0 in the classification of symptom groups, which again confirms that 1.5 is a better cutoff point for the CCQ. 
Table 8 Spearman rank correlations between health status questionnaires (CAT and CCQ), lung function, exacerbation frequency in the previous year, and $m M R C$ dyspnea scale at baseline $(n=372)$

\begin{tabular}{|c|c|c|c|c|c|c|c|c|}
\hline Variables & CAT & $\begin{array}{l}\text { CCQ } \\
\text { total }\end{array}$ & $\begin{array}{l}\text { CCQ } \\
\text { symptom }\end{array}$ & $\begin{array}{l}\text { CCQ } \\
\text { function }\end{array}$ & $\begin{array}{l}\text { CCQ } \\
\text { mental }\end{array}$ & mMRC & $\begin{array}{l}\mathrm{FEV}_{1} \% \\
\text { predicted }\end{array}$ & $\begin{array}{l}\text { Exacerbation } \\
\text { frequency }\end{array}$ \\
\hline CAT & - & 0.776 ** & $0.590 * *$ & $0.688 * *$ & $0.409 * *$ & $0.614 * *$ & $-0.270 * *$ & 0.516 ** \\
\hline CCQ total & - & - & $0.734 * *$ & $0.829 * *$ & $0.628 * *$ & $0.540 * *$ & $-0.27 \mid * *$ & $0.542 * *$ \\
\hline CCQ symptom & - & - & - & $0.497 * *$ & $0.273 * *$ & $0.346 * *$ & $-0.27 \mid * *$ & $0.439 * *$ \\
\hline CCQ function & - & - & - & - & $0.334 * *$ & $0.578 * *$ & $-0.321 * *$ & $0.468 * *$ \\
\hline CCQ mental & - & - & - & - & - & $0.284 * *$ & $-0.132 *$ & $0.339 * *$ \\
\hline $\mathrm{mMRC}$ & - & - & - & - & - & - & $-0.315 * *$ & $0.342 * *$ \\
\hline $\mathrm{FEV}, \%$ predicted & - & - & - & - & - & - & - & $-0.280 * *$ \\
\hline Exacerbation frequency & - & - & - & - & - & - & - & - \\
\hline
\end{tabular}

Notes: $* * P<0.01, * P<0.05$.

Abbreviations: CAT, COPD Assessment Test; CCQ, Clinical COPD Questionnaire; FEV , forced expiratory volume in I second; mMRC, modified Medical Research Council dyspnea score.

Our study demonstrated that the CAT and CCQ correlated well with each other and both were able to clearly distinguish between patients with different COPD severity assessed by exacerbation rates, $\mathrm{FEV}_{1} \%$ predicted, and mMRC. With higher scores of the CAT and CCQ, subjects displayed more impairment in lung function, higher level of mMRC, and greater number exacerbations in the last year. Similarly, patients with more exacerbations presented worse scores on the CAT and total CCQ as well as its 3 domains, which suggested that the CAT and CCQ might be good indicators of exacerbation frequencies. Other studies also support our findings: both these health status questionnaires have proved to be associated with exacerbations ${ }^{11}$ and CAT has been identified as a strong predictor of the composite events in COPD. ${ }^{12-15}$ Miravitlles et $\mathrm{a}^{16}$ found that scores of both the CAT and CCQ significantly decreased when subjects recovered from exacerbations. However, in our study no obvious differences were detected in the score of the CAT, and the

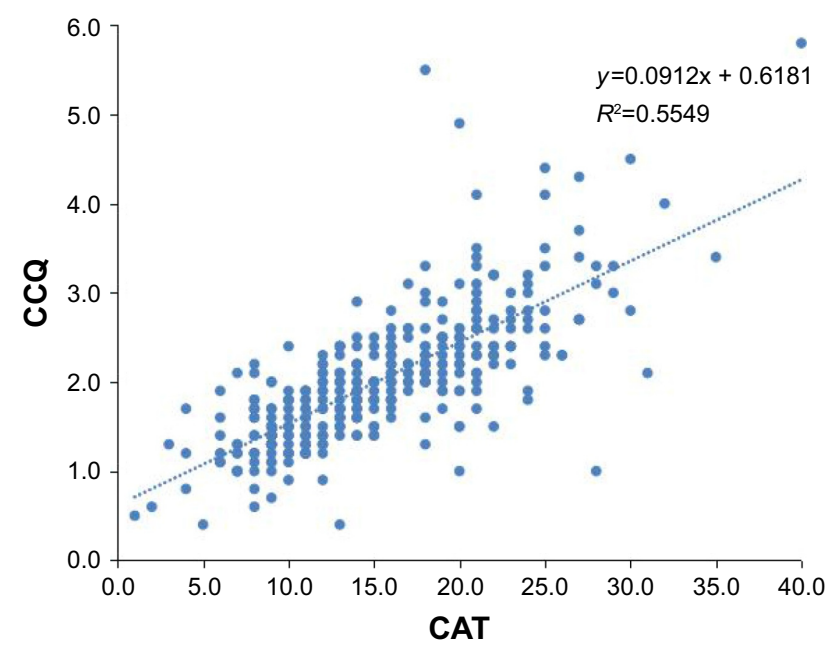

Figure 4 Spearman rank correlations between the CAT and the total CCQ score. Abbreviations: CAT, COPD Assessment Test; CCQ, Clinical COPD Questionnaire. total CCQ as well as its 3 domains between grades 1 and 2 . In addition, only weak correlation was obtained between the 2 tools and $\mathrm{FEV}_{1} \%$ predicted. These results were in keeping with Jones ${ }^{17}$ and Josephs ${ }^{18}$ studies. We also found that both the CAT and CCQ had a much stronger correlation with the number of exacerbations in the last 12 months than $\mathrm{FEV}_{1} \%$ predicted. COPD is a heterogeneous disease with different clinical phenotypes both in the stable phase and in acute exacerbations, ${ }^{19,20}$ and a variety of risk factors are associated with exacerbations. ${ }^{21,22}$ Therefore, prediction of $\mathrm{FEV}_{1} \%$ alone cannot accurately reflect the full impact of COPD on daily life.

Limitations to this study include the following: first, this study was limited to 1 single center; second, this was a cross-sectional study without any analysis of prospective data on exacerbations. A further study should focus on a comparison of predictive value of the $\mathrm{CAT}, \mathrm{CCQ}, \mathrm{FEV}_{1} \%$ predicted, and exacerbation history for the future exacerbations in COPD.

\section{Conclusion}

As comprehensive health status measures, both the CAT and the CCQ were proposed by GOLD. In comparison with the CAT, the CCQ was more likely to place patients into more severe categories, and 1.5 is a more appropriate cutoff point for the CCQ than 1.0. Both the CAT and the overall CCQ with its 3 domains could clearly discriminate between patients with different COPD severity.

\section{Acknowledgment}

This study was funded by the National Natural Science Foundation of China (81370143 to Ping Chen) and the Fundamental Research Funds for the Central Universities of Central South University (2016zzts144 to Zijing Zhou). 


\section{Author contributions}

$\mathrm{PC}$ and $\mathrm{ZZ}$ contributed to the study design; ZZ contributed to the drafting of the manuscript; $P C$ contributed to revising it critically for important intellectual content. ZZ, AZ, YZ, and JD contributed to data collection. All authors contributed toward data analysis, drafting and revising the paper and agree to be accountable for all aspects of the work.

\section{Disclosure}

The authors report no conflicts of interest in this work.

\section{References}

1. Global Initiative for Chronic Obstructive Lung Disease (GOLD). Global Strategy for the Diagnosis, Management, and Prevention of Chronic Obstructive Pulmonary Disease. 2011.

2. Global Initiative for Chronic Obstructive Lung Disease (GOLD). Global Strategy for the Diagnosis, Management, and Prevention of Chronic Obstructive Pulmonary Disease. 2013.

3. Jones PW, Harding G, Berry P, Wiklund I, Chen WH, Kline Leidy N. Development and first validation of the COPD Assessment Test. Eur Respir J. 2009;34(3):648-654.

4. van der Molen T, Willemse BW, Schokker S, ten Hacken NH, Postma DS, Juniper EF. Development, validity and responsiveness of the clinical COPD questionnaire. Health Qual Life Outcomes. 2003;1:13.

5. Kon SS, Canavan JL, Nolan CM, et al. The clinical chronic obstructive pulmonary disease questionnaire: cut point for GOLD 2013 classification. Am J Respir Crit Care Med. 2014;189(2):227-228.

6. Tsiligianni IG, Kocks JW, van der Molen T. Highlighting CCQ-CAT calculation and advantages. COPD. 2012;9(3):316-317.

7. Landis JR, Koch GG. The measurement of observer agreement for categorical data. Biometrics. 1977;33(1):159-174.

8. Yohannes AM, Alexopoulos GS. Depression and anxiety in patients with COPD. Eur Respir Rev. 2014;23(133):345-349.

9. Pooler A, Beech R. Examining the relationship between anxiety and depression and exacerbations of COPD which result in hospital admission: a systematic review. Int J Chron Obstruct Pulmon Dis. 2014;9:315-330.

10. Panagioti M, Scott C, Blakemore A, Coventry PA. Overview of the prevalence, impact, and management of depression and anxiety in chronic obstructive pulmonary disease. Int J Chron Obstruct Pulmon Dis. 2014;9:1289-1306.
11. Sundh J, Ställberg B, Lisspers K, Kämpe M, Janson C, Montgomery S. Comparison of the COPD Assessment Test (CAT) and the ClinicalCOPD Questionnaire (CCQ) in a clinical population. COPD. 2016; 13(1):57-65.

12. Varol Y, Ozacar R, Balci G, Usta L, Taymaz Z. Assessing the effectiveness of the COPD Assessment Test (CAT) to evaluate COPD severity and exacerbation rates. COPD. 2014;11(2):221-225.

13. Lee SD, Huang MS, Kang J, et al; Investigators of the Predictive Ability of CAT in Acute Exacerbations of COPD (PACE) Study. The COPD assessment test (CAT) assists prediction of COPD exacerbations in high-risk patients. Respir Med. 2014;108(4):600-608.

14. Miravitlles M, García-Sidro P, Fernández-Nistal A, et al. The chronic obstructive pulmonary disease assessment test improves the predictive value of previous exacerbations for poor outcomes in COPD. Int J Chron Obstruct Pulmon Dis. 2015;10:2571-2579.

15. García-Sidro P, Naval E, Martinez Rivera C, et al. The CAT (COPD Assessment Test) questionnaire as a predictor of the evolution of severe COPD exacerbations. Respir Med. 2015;109(12):1546-1552.

16. Miravitlles M, García-Sidro P, Fernández-Nistal A, Buendía MJ, Espinosa de los Monteros MJ, Molina J. Course of COPD assessment test (CAT) and clinical COPD questionnaire (CCQ) scores during recovery from exacerbations of chronic obstructive pulmonary disease. Health Qual Life Outcomes. 2013;11:147.

17. Jones PW, Brusselle G, Dal Negro RW, et al. Properties of the COPD assessment test ina cross-sectional European study. Eur Respir J. 2011; 38(1):29-35.

18. Joseph S, Pascale S, Georges K, Mirna W. Cigarette and waterpipe smoking decrease respiratory quality of life in adults: results from a national cross-sectional study. Pulm Med. 2012;2012:868294.

19. Lange P, Halpin DM, O'Donnell DE, MacNee W. Diagnosis, assessment, and phenotyping of COPD: beyond $\mathrm{FEV}_{1}$. Int J Chron Obstruct Pulmon Dis. 2016;11 Spec Iss:3-12.

20. Zhou A, Zhou Z, Zhao Y, Chen P. The recent advances of phenotypes in acute exacerbations of COPD. Int J Chron Obstruct Pulmon Dis. 2017; 12:1009-1018.

21. Müllerova H, Maselli DJ, Locantore N, et al. Hospitalized exacerbations of COPD: risk factors and outcomes in the ECLIPSE cohort. Chest. 2015;147(4):999-1007.

22. Alexopoulos EC, Malli F, Mitsiki E, Bania EG, Varounis C, Gourgoulianis KI. Frequency and risk factors of COPD exacerbations and hospitalizations: a nationwide study in Greece (Greek Obstructive Lung Disease Epidemiology and health ecoNomics: GOLDEN study). Int J Chron Obstruct Pulmon Dis. 2015;10:2665-2674.
International Journal of COPD

\section{Publish your work in this journal}

The International Journal of COPD is an international, peer-reviewed journal of therapeutics and pharmacology focusing on concise rapid reporting of clinical studies and reviews in COPD. Special focus is given to the pathophysiological processes underlying the disease, intervention programs, patient focused education, and self management protocols.

\section{Dovepress}

This journal is indexed on PubMed Central, MedLine and CAS. The manuscript management system is completely online and includes a very quick and fair peer-review system, which is all easy to use. Visit $\mathrm{http}: / / \mathrm{www}$.dovepress.com/testimonials.php to read real quotes from published authors. 